Ebisu Ebisu

Études japonaises Études japonaises

47 | printemps-été 2012

Catastrophes du 11 mars 2011, désastre de

Fukushima : fractures et émergences

\title{
Enjeux de la reconstruction : recoudre le tissu urbain, renouer le lien social
}

Challenges to Post-Disaster Reconstruction: Repairing the Urban Fabric and Reestablishing Social Cohesion

復興の諸問題一都市という織布をつくろい直す、社会的紐带を結び 直す

\section{Murielle Hladik}

\section{OpenEdition}

Journals

Édition électronique

URL : http://journals.openedition.org/ebisu/436

DOI : $10.4000 /$ ebisu.436

ISSN : 2189-1893

Éditeur :

Institut français de recherche sur le Japon (UMIFRE 19 MAEE-CNRS), Maison franco-japonaise

Édition imprimée

Date de publication : 1 juin 2012

Pagination : 189-203

ISSN : 1340-3656

Référence électronique

Murielle Hladik, «Enjeux de la reconstruction : recoudre le tissu urbain, renouer le lien social », Ebisu [En ligne], 47 | printemps-été 2012, mis en ligne le 21 mai 2014, consulté le 01 mai 2019. URL : http:// journals.openedition.org/ebisu/436 ; DOI : 10.4000/ebisu.436 


\title{
Enjeux de la reconstruction \\ Recoudre le tissu urbain, renouer le lien social
}

\author{
Murielle HLADIK
}

Quels sont les enjeux éthiques, économiques, environnementaux et sociaux de la reconstruction? Après le grand tremblement de terre de l'Est du Japon, ces enjeux sont de taille pour les architectes, les bureaux de constructeurs et les urbanistes. Après la phase de l'intervention d'urgence, visant à reloger les populations sans logement et, dans un premier temps, à trouver des solutions de fortune, va venir la phase post-catastrophe de la reconstruction. Se pose alors, sur ces terrains par nature précaires, la question de la sécurité : vis-à-vis de la menace d'un nouveau tremblement de terre, de la menace des tsunamis et, surtout, de cette menace radicalement " nouvelle " des terres contaminées. Force est de dire que les enjeux environnementaux ne sont pas du tout de même nature : dans toute l'histoire de l'archipel nippon, les catastrophes naturelles ont été pensées dans les consciences collectives, les risques - dans une certaine mesure - calculés, et les contrôles d'ingénierie, les calculs des risques sismiques ajustés en conséquence. Si les calculs antisismiques sont très performants au Japon, face aux tsunamis et à la force des océans, néanmoins, comme on a pu le voir, malgré toute l'ingéniosité des architectes et des ingénieurs, la plupart des petites habitations ont été littéralement balayées. Quant à la menace nucléaire - c'est-à-dire la réalité actuelle des sols, des terres et des eaux contaminés pour de nombreuses

Murielle Hladik, architecte DPLG et docteur en philosophie, est chercheure associée (Maison des Sciences de l'Homme - Paris-Nord) et membre du réseau Japarchi. Depuis mars 2011, elle est administratrice de l'association Patrimoine sans frontières. 


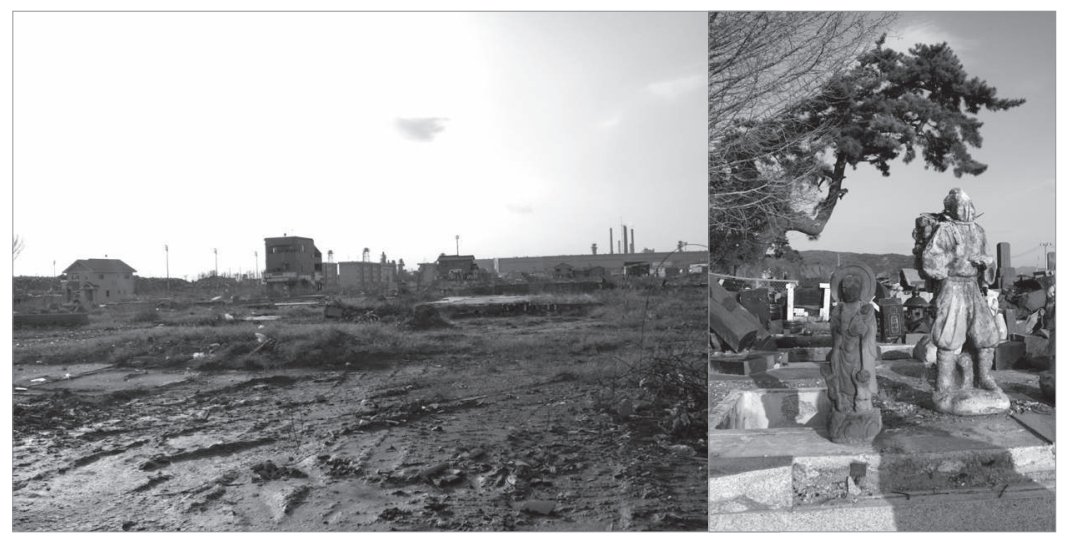

Fig. 1 et 2 Ishinomaki $\odot$ Murielle Hladik

années - elle n’a pas été pensée en tant que telle et surtout elle n’a pas été anticipée par les pouvoirs publics qui ont plutôt minimisé les risques.

\section{Un tissu urbain disloqué - Reconstruire = recoudre}

Des architectes, des sociologues et des urbanistes ont créé l'association Archi+Aid, en lien avec la Sendai Design League, qui vise à réunir un collectif de réflexion sur les enjeux non seulement architecturaux mais aussi éminemment sociaux de la reconstruction ${ }^{1}$. Comment reconstruire sur des terrains qui ne seront pas potentiellement immergés par les tsunamis (digues, construction en hauteur, etc.), mais surtout comment recoudre le lien social qui existait auparavant dans les villages de pêcheurs aujourd'hui dévastés ? Peut-on envisager un " risque zéro " ? Comment se pose la question d'une identité collective dans ce contexte?

Toute la baie d'Ishinomaki 石巻 a été ravagée par le tsunami : 19360 maisons totalement détruites ; 3949 maisons partiellement détruites ;

1. Archi+ Aid. Relief an Recovery by Architects for Tohoku Earthquake and Tsunami : $<$ http://archiaid.org/>. 


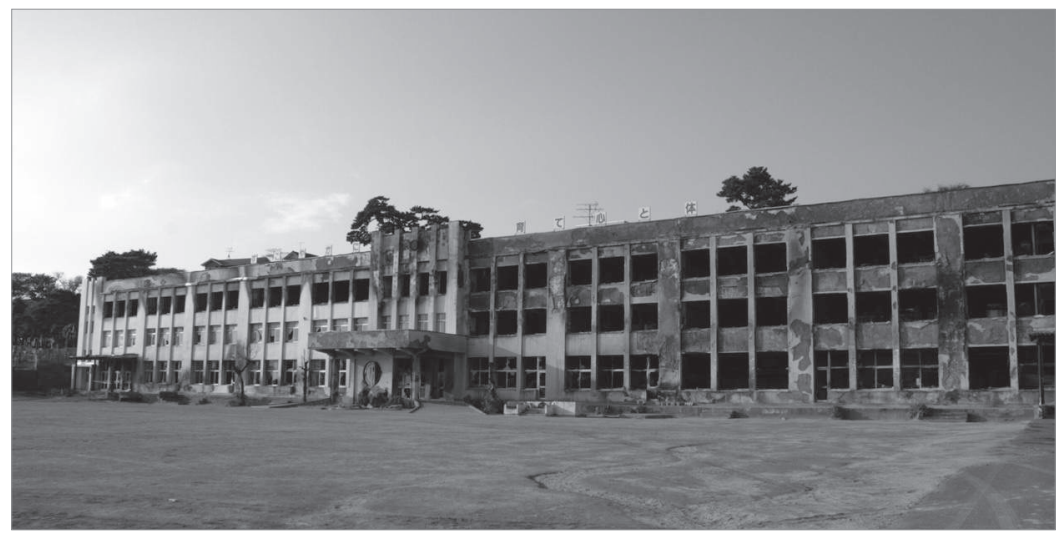

Fig. 3 École élémentaire de Kadonowaki @ MH

9466 maisons partiellement endommagées ${ }^{2}$. En novembre 2011, je rencontre Matsumura Yoshiyuki 松村善行, le directeur de l'association locale Support Ishinomaki ${ }^{3}$. Nous parcourons ensemble les lieux dévastés; le déblaiement a maintenant en grande partie été effectué (fig. 1 et 2). Aux abords du pont, des ouvriers sur une drague essaient de dégager les restes de carcasses de voitures et de bateaux qui ont été submergés. Le paysage est radicalement vide, des montagnes de détritus et de carcasses rouillées de voitures indiquent le travail effectué. Ici, un temple dévasté dont il ne reste que l'ossature, le moine est décédé - m’annonce sobrement Matsumura. Plus loin, des tombes dévastées...

Devant l'emplacement de ce qui était sa propre maison, Matsumura éclate de rire et déclare : "My home ". Il me demande ce que je pense en tant qu'architecte des nouvelles lois que les urbanistes essaient de mettre en place pour créer une zone non-aedificandi dans tout le secteur potentiellement inondable. Vif débat : les architectes et les urbanistes discutent de la nécessité de créer cette zone, tandis que les habitants seraient prêts à revenir habiter sur les lieux. Ce désir de revenir habiter en ces lieux, malgré

2. Source : Nippon Design Center, <http://311scale.jp/>.

3. NPO Support Ishinomaki : <http://www.i-support.or.jp/>. 


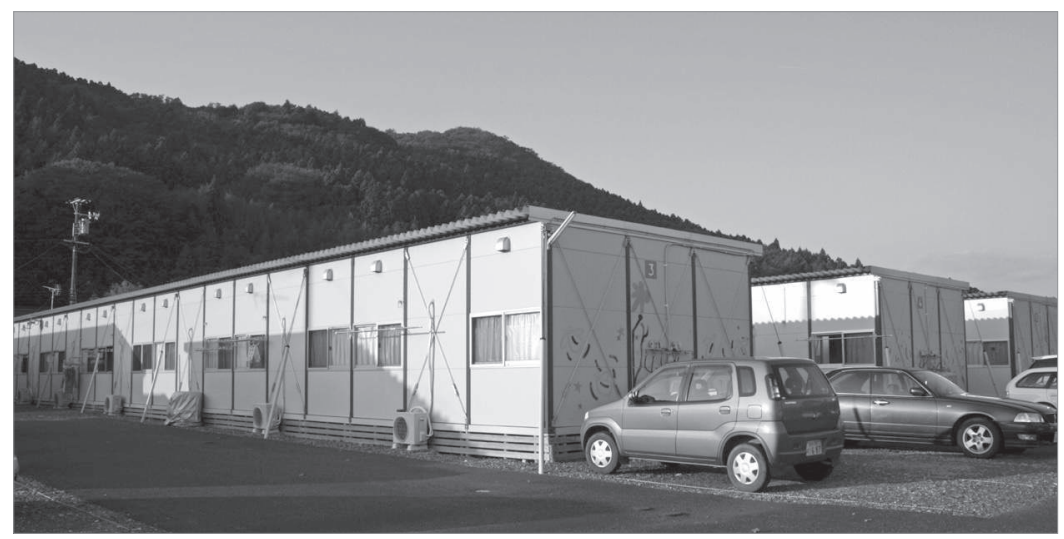

Fig. 4 Ishinomaki, habitations provisoires (c) $\mathrm{MH}$

toute la force du désastre, malgré l'intensité du vécu parait, pour un regard occidental, complètement démesuré.

Nous allons voir la mer. Elle est toujours là, avec son flux et son reflux permanent. De là vient le danger, mais comment l'éviter ? Faut-il, comme le suggèrent les architectes et les urbanistes, construire face à la mer une autoroute surélevée à huit mètres de hauteur permettant une substantielle protection contre sa violence dévastatrice ? Les rangées de tétrapodes de béton placées dans la mer nous paraissent, aujourd'hui, bien dérisoires.

Au fond de la baie, se dresse l'école élémentaire de Kadonowaki (Ishinomaki shiritsu Kadonowaki shōgakkō 石巻市立門脇小学校), vaste édifice de béton sur huit étages qui a été en partie carbonisé (fig. 3). Les rares survivants ayant réussi à échapper au désastre se sont réfugiés là, en hauteur, pour s'abriter des vagues ; ensuite l'incendie s'est déclaré. Les survivants ont dû se réfugier plus loin dans les montagnes. L'école est devenue un lieu de mémoire, non seulement pour les enfants mais aussi pour les familles. Devant cette école, l'association Support Ishinomaki organise des événements collectifs autour de la mémoire, des jeux pour les enfants et surtout des événements sportifs.

Après la phase d'urgence, où des édifices publics avaient été réquisitionnés, les habitants ont été relogés dans des habitations provisoires (kasetsu jūtaku 仮設住宅) ; pour la seule ville d'Ishinomaki, 4000 personnes sont 


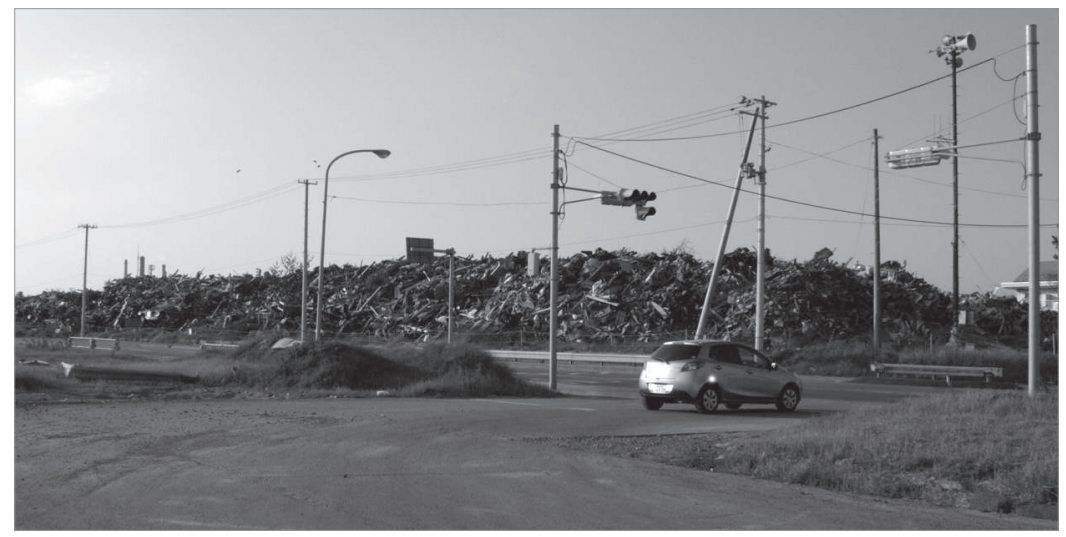

Fig. 5 Ishinomaki, montagnes de débris $\odot \mathrm{MH}$

encore, au mois de novembre 2011, logées dans ces habitations (fig. 4) sur un seul niveau $(R+1)$, des containers alignés à la file les uns derrière les autres. C'est un paysage rude. Les habitations provisoires sont placées un peu à l'écart du centre ville. Si la vie collective commence peu à peu à s'organiser, le quotidien demeure rudimentaire.

De plus, à proximité, les montagnes de débris qui ont été ramassés, encore en attente de traitement, posent des questions sanitaires et dégagent des odeurs nauséabondes (fig. 5). Le traitement de ces déchets constitue un autre grand sujet de préoccupation - surtout en ce qui concerne le traitement des déchets contaminés. Huit mois après le séisme, le recyclage des masses de détritus constitue un réel souci pour la communauté. Avec près de 6 millions de tonnes de débris pour la seule ville d'Ishinomaki ${ }^{4}$, le traitement n'est plus réalisable par le seul département de Miyagi. Si le département de Tokyo a accepté de traiter et d'incinérer une partie de ces déchets, la question du risque de contamination reste préoccupante pour l'ensemble des autres départements.

4. Voir journal Mainichi : <http://mdn.mainichi.jp/features/news/20111113p2g00m0 fe033000c.html>. 
De retour au quartier général de l'association, je pose la question de savoir quel serait leur souhait pour la reconstruction ? Le mot clé de l'association est «Gambare Ishinomaki »がんばれ石巻 : c'est-à-dire, tous ensemble, on va mettre toute notre énergie pour essayer de surmonter les difficultés ${ }^{5}$. La demande majeure qui émerge est celle d'un lieu communautaire où les enfants pourraient jouer. Si les habitations provisoires ont été conçues dans l'urgence et, en premier lieu, pour les adultes, l'absence de lieux collectifs conçus pour les enfants engendre une réelle demande de la part des habitants. Le projet souhaité par les habitants serait une maison de quartier, un lieu de jeu pour les enfants : un espace communautaire.

\section{Identité et mémoire collective}

Après la phase de déblaiement des débris, la question de la sauvegarde du patrimoine - de ce qui doit être préservé ou non - commence à émerger. Après l'effacement des traces, se pose la délicate question de la mémoire et de l'oubli.

Igarashi Tarō 五十嵐太郎, critique d'architecture, professeur à l'université du Tōhoku, et également membre de l'association Archi+Aid, me fait part des réflexions sur la mémoire de la catastrophe et la conservation de certains bâtiments menées dans le cadre de son université. La question se pose notamment à propos d'un édifice en béton totalement renversé dans la ville dévastée d'Onagawa 女川町 (fig. 6). La question du recueil de la mémoire orale des survivants et de leur vécu est également à l'ordre du jour.

Faut-il construire en hauteur? Comment reconstituer une infrastructure sur des kilomètres de côtes dévastées? Les habitants se sont réfugiés dans les constructions en hauteur en béton qui ont, dans la plupart des cas, mieux résisté au tsunami. L'habitat pavillonnaire horizontal qui constitue la majorité du paysage urbain nippon a lui été massivement emporté par les flots.

5. Le manque de travail et la récession économique vont être pour les prochaines années les problèmes majeurs de cette région sinistrée. Le magasin de sport où nous nous trouvons, transformé en QG de l'association, a été miraculeusement préservé, mais la plupart des autres boutiques ont été dévastées, laissant les rez-de-chaussée et les étals des marchands désespérément vides. 
Si la construction antisismique est bien développée au Japon, la violence des flots a littéralement balayé les fondations de ces petites habitations. Une des options proposées par les architectes est de construire, de loin en loin, des structures verticales qui serviraient d'abri et de refuge à la collectivité en cas d'alerte et de tsunamis ${ }^{6}$.

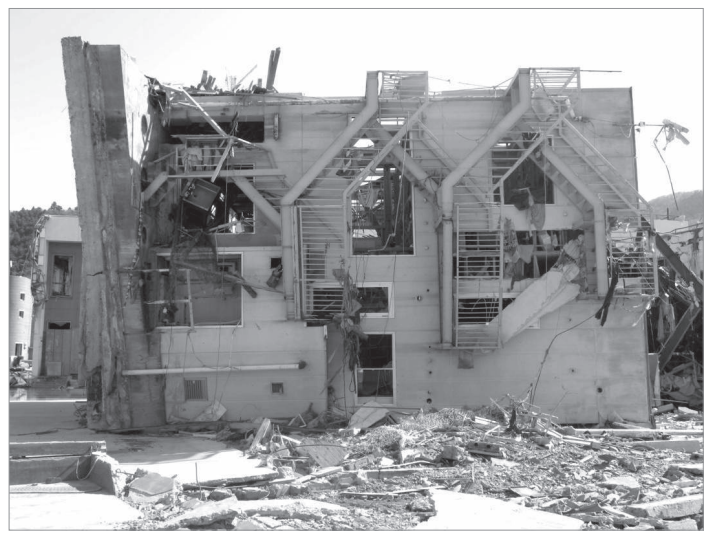

Fig. 6 Ville d'Onagawa @ Igarashi Tarō Laboratory

Enfin, plus fondamentalement, l'association des architectes japonais Archi+Aid réfléchit à la construction d'une " maison pour tous " (minna no ie みんなの家). L'architecte Itō Toyoo 伊東豊雄, en collaboration avec les jeunes architectes Inui Kumiko 乾久美子, Fujimoto Sōsuke 藤本壮介 et Hirata Akihisa 平田晃久, a lancé un appel afin de concevoir cette «maison pour tous " répondant aux exigences de la reconstruction et afin de redonner un sens dans l'organisation de la vie sociale. L'ensemble de ces projets - 900 dessins d'architectes et d'étudiants du monde entier - sera exposé lors de la prochaine Biennale d'architecture de Venise (2012). Le premier prototype de "maison pour tous " a été réalisé dans la ville de Sendai, fin octobre 2011 (fig. $7 \mathrm{a} / \mathrm{b} / \mathrm{c} / \mathrm{d}$ ).

6. Des « refuges » en hauteur, à l'image des rochers des anachorètes, des ermites ou des saints (hijiri 聖) qui se réfugiaient dans les montagnes. 


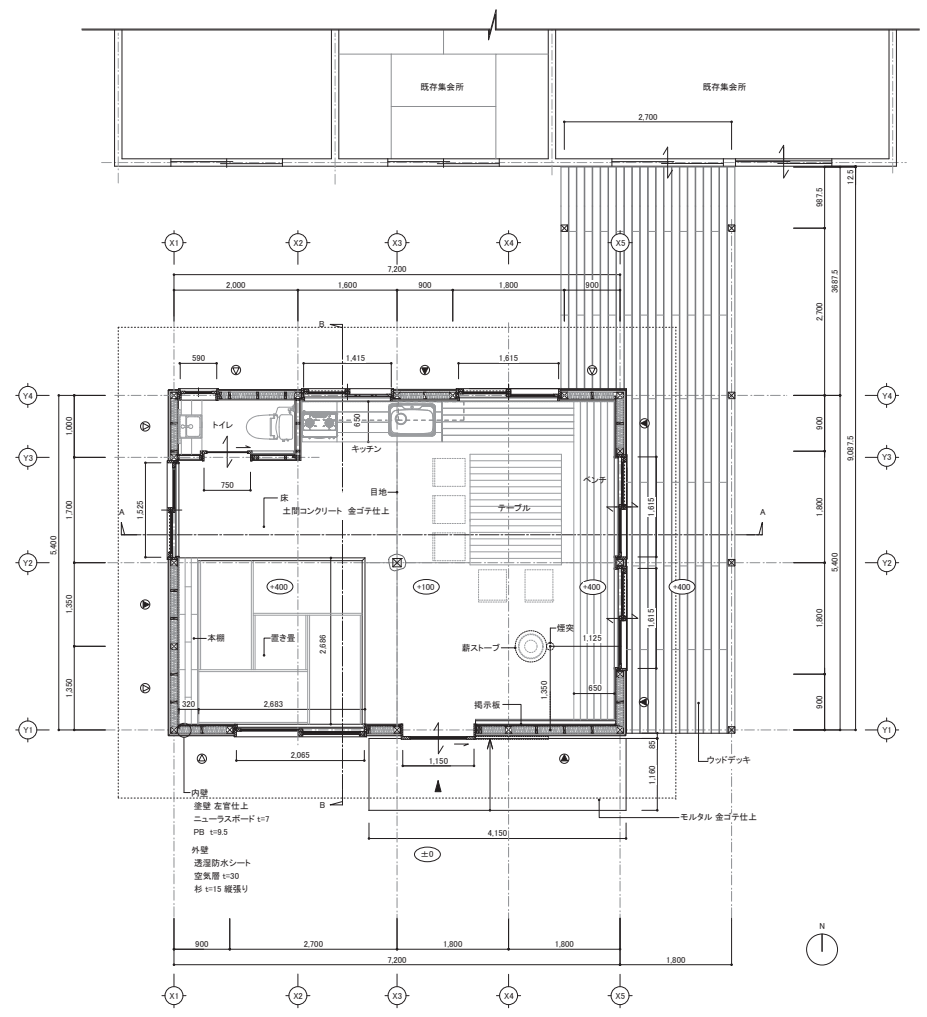

平面図 $S=1: 50$

4 : 肪通 $45 \times 90$ タัフル

20111108

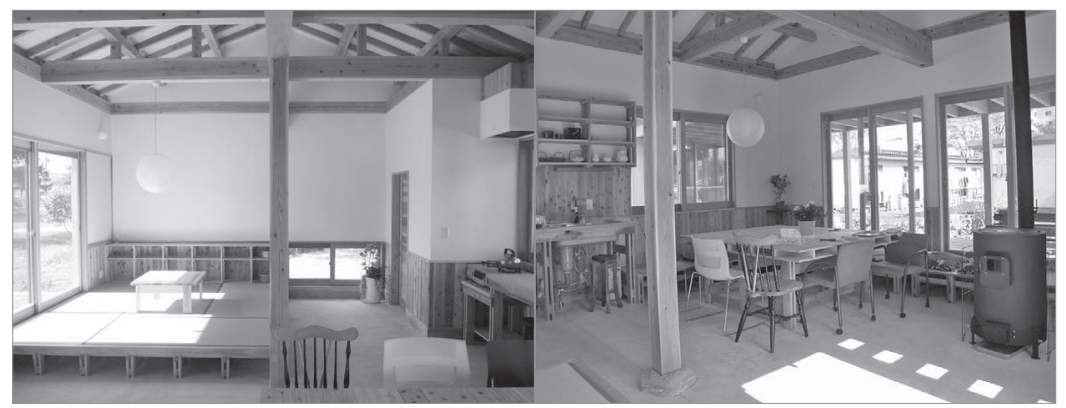




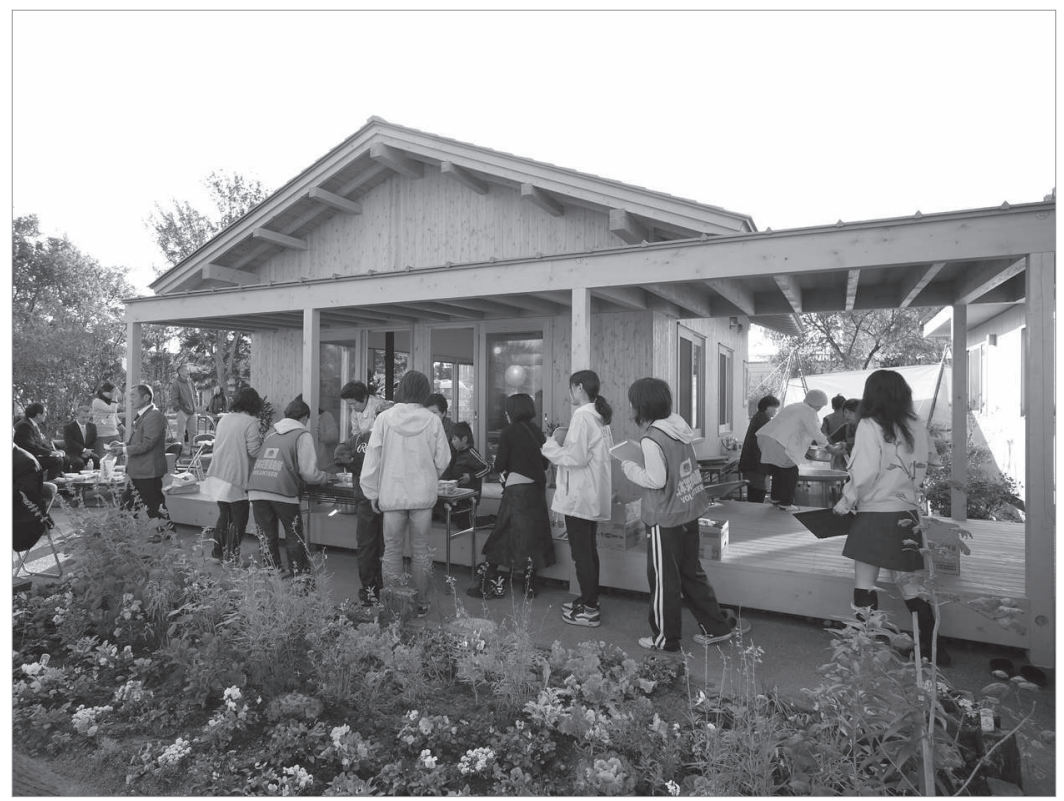

Fig. 7 a/b/c/d (ci-contre et ci-dessus) Itō Toyoo, avec Inui Kumiko, Fujimoto Sōsuke et Hirata Akihisa, "Maison pour tous », premier prototype réalisé à Sendai avec la participation active des résidents (C) Itō Toyoo

Lors du symposium qui s'est tenu à l'université de Tokyo, le 2 novembre 2011, des architectes se sont interrogés sur le statut de l'architecture et les potentialités de la reconstruction ${ }^{7}$. Itō Toyoo présentait le projet de "Maison pour tous» (minna no ie みんなの家) dans la quatrième partie intitulée : «Renaissance : les potentialités de l'architecture » (Saisei : kenchiku ni dekiru koto 再生:建築にできること) ${ }^{8}$. C'est-à-dire l'idée d'une renaissance,

7. Voir : <http://www.toto.co.jp/gallerma/ex111102/sympsm.htm>.

8. Itō Toyoo déclarait :

"Several months after the massive earthquake and tsunami hit the Tohoku coast in Eastern Japan, calm seas and lush vegetation have finally returned to the devastated floodplains. As spring green began appearing among the rubble and the sounds of songbirds were heard again, so human activity, too, resumed, making way amid the debris for the resumption of normal 
d'une régénération (saisei 再生) et d'une reconstruction (tatenaosu 立て直す) des bâtiments et d'autres édifices, ainsi que de ce qui constitue le lien entre les hommes : c'est-à-dire la reconstruction du tissu social.

Ce symposium fut organisé à l'occasion de l'inauguration de l'exposition « 311 Lost Homes » à la galerie Ma 間 à Tokyo ${ }^{9}$, réalisée avec le soutien de l'association Archi+Aid, de la Japan Association of Architectural Firms, du Japan Institute for Architects, de la Sendai Design League, réunissant la plupart des grandes universités japonaises. Le parti pris des commissaires de l'exposition, Naitō Hiroshi 内藤廣 et Hara Ken.ya 原研哉, fut la représentation de "fragments urbains » : de grandes maquettes blanches du paysage urbain " avant la vague ", de ces 311 maisons aujourd'hui disparues. Le parti pris de l'ex-position - de ce qui est mis en lumière, "jeté-là " - est volontairement présenté avec une certaine neutralité et froideur : la mémoire du paysage disparu est simplement représentée en blanc. Des photographies de ces " fragments " par Google Earth avant/après montrent l'étendue des dégâts. Sur les murs sont affichés les cartes et les plans de cette portion de territoire ravagée par le tsunami sur près de $300 \mathrm{~km}$ de linéaire côtier. Les zones dévastées sont représentées en rouge-rosé, tandis que les chiffres des destructions et les seuils de radiations sont décrits minutieusement à l'aide de graphes. Le texte de présentation invite les visiteurs à la réflexion et au calme : de l'ordre de l'indicible, du "sans-voix ». Le parti pris de l'exposition n'est pas de représenter directement le vide et le désastre laissé par la catastrophe, ni même des propositions pour une future reconstruction - ce qui est peut-être, neuf mois après la catastrophe, encore prématuré -, mais plus sobrement, le paysage urbain " avant la vague ». Si les architectes ont sou-

activities. In their impetuous efforts to rebuild homes and work places from scratch, we are witnessing the determination and power of people's primal instinct to survive and carry on with their lives. In that energy we cannot help sensing the vitality of "building" and "living", the fundamental meaning of which we had nearly forgotten in the course of ordinary daily life before the disaster. " (<http://www.designboom.com/weblog/cat/9/view/17528/architecturein-the-wake-of-disaster-japan-pavilion-for-the-2012-venice-architecture-biennale.html>).

Sur cette question d'une potentielle régénération par les plantes, je renvoie au symposium dont j'ai assuré la co-organisation scientifique : "Architecture et nature : renaissance " (Kenchiku to shizen : saisei 建築と自然——再生), Institut franco-japonais du Kansai, le 21 avril 2012.

9. Exposition “ 311 Lost Homes " (San ichi ichi ushinawareta machi 311 失われた街, du 2 novembre au 24 décembre 2011 : <http://www.toto.co.jp/gallerma/>. 
haité cette sobriété dans la représentation, c'est aussi en concertation avec les habitants ${ }^{10}$, comme si ceux-ci, avant de pouvoir envisager une potentielle reconstruction, avaient besoin de se remémorer le paysage d'avant. Comme si un équilibre entre mémoire et oubli était nécessaire afin de pouvoir faire le « deuil » de ce qui a été détruit et radicalement effacé.

\section{Mémoire du paysage et intervention des artistes}

C'est l'ensemble du paysage social, économique et mental qui a été bouleversé. La réaction des artistes, des plasticiens et des photographes a, elle aussi, été très forte. Peut-être les artistes sont-ils les plus à même de pointer du doigt les enjeux éthiques et de porter un regard critique sur les conflits d'intérêt que va nécessairement soulever la reconstruction. Des initiatives locales d'entraide et de soutien se mettent en place. La plupart des artistes ont d'abord connu une phase de non production ; après un arrêt total, ils ont ensuite redémarré leurs œuvres, le plus souvent avec un travail critique. Le photographe Hatakeyama Naoya 畠山直哉 a traversé les paysages dévastés au lendemain du tsunami. Originaire de Rikuzentakata 陸前高田, une ville portuaire de 23000 habitants presque entièrement détruite par le séisme et le tsunami, le paysage de son enfance ravagé par la vague nous bouleverse. En novembre 2011, je rencontre Hatakeyama Naoya et nous discutons autour de la notion de distanciation et de « détachement du regard"(riken no ken 離見の見 $)^{11}$. Sa dernière exposition "Natural Stories » (Tokyo Metropolitan Museum of Photography) est une

10. Symposium «Ville et architecture après le 11 mars. Comment les architectes régénèrent-ils le local ?" (San ten ichi ichi go no kenchiku, toshi. Kenchikuka tachi wa chiiki $o$ dō saisei shiyō to shiteiru no ka? 3.11 後の建築・都市——建築家たちは地域をどう再生しよ うとしているのか), Maison de la Culture du Japon à Paris et École nationale supérieure d'architecture de Paris-Malaquais (29-31 mars 2012).

11. Cette notion centrale de l'esthétique japonaise a été développée par le dramaturge Zeami 世阿弥 (1363-1443). Selon Hatakeyama, toute photographie contient de manière intrinsèque cet idéal d'un détachement. Au-delà de cette problématique de la distanciation, la question que pose le présent travail photographique de Hatakeyama Naoya est également celle de la légitimité de la représentation : faut-il représenter l'irreprésentable? 


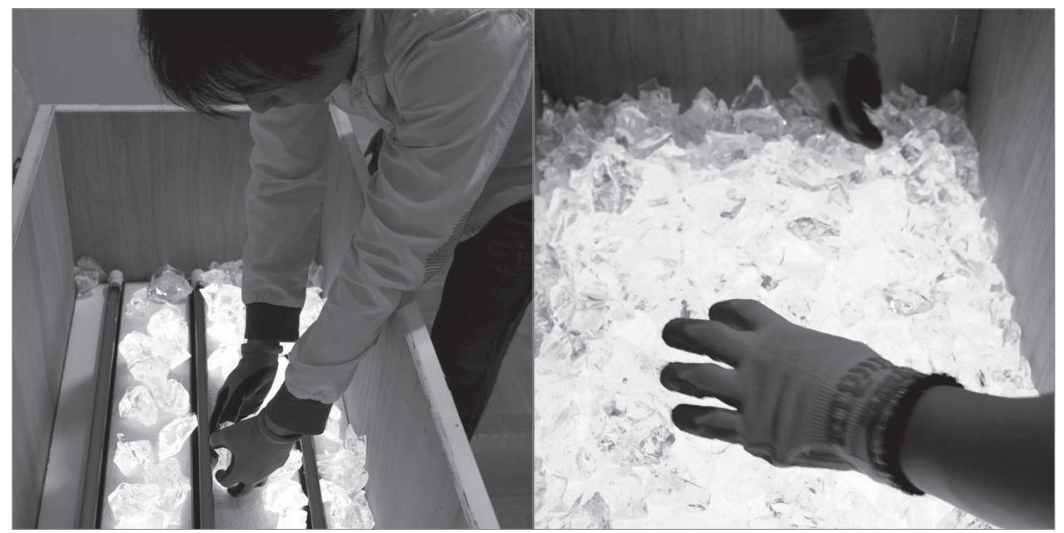

Fig. 8 a/b Kondō Takahiro, installation Hotaru (uranium et verre concassé), exposition « Architecture et impermanence » (Kenchiku to mujō 建築と無常), Institut franco-japonais du Kansai, avril 2012. Photos de montage : caisse contenant des morceaux de verre concassé @ Ana Amelia Genioli.

synthèse de son œuvre au cours de ces dernières années. Mettant en écho les photos de la destruction avec des scènes plus paisibles du paysage avant la catastrophe, Hatakeyama interroge la notion de sublime : c'est-à-dire la part tragique que constitue la perte et finalement, au-delà des mots et de la représentation, la violence et la beauté de la nature.

Le céramiste Kondō Takahiro 近藤高弘 a réalisé une série d'installations intitulée Hotaru 20000 dédiée à la mémoire des 20000 disparus (fig. 8 $\mathrm{a} / \mathrm{b} / \mathrm{c}$ ). Il utilise des morceaux de verre dans lesquels est incorporé de l'uranium. À première vue, ces plaques paraissent être de simples conglomérats de morceaux de verre, mais éclairées avec une lumière noire, l'uranium qu'elles contiennent émet un signal lumineux et elles brillent dans la nuit à la manière des lucioles (d'où le nom de hotaru 蛍). Ces pièces interrogent le spectateur sur le caractère invisible des radiations et sur le danger impalpable et incontrôlable qu'elles constituent. Dans un autre projet dédié au Tōhoku, Kondō œuvre avec un collectif d'artistes céramistes qui fabriquent des bols dont ils font ensuite don aux habitants de la région. Le bol ou " réceptacle» (utsuwa うつわ/器) dépasse le simple " contenant » et renvoie dans toute sa dimension philosophique aux moines bouddhistes qui font l'aumône de leur nourriture, ne possédant rien d'autre qu'un simple 


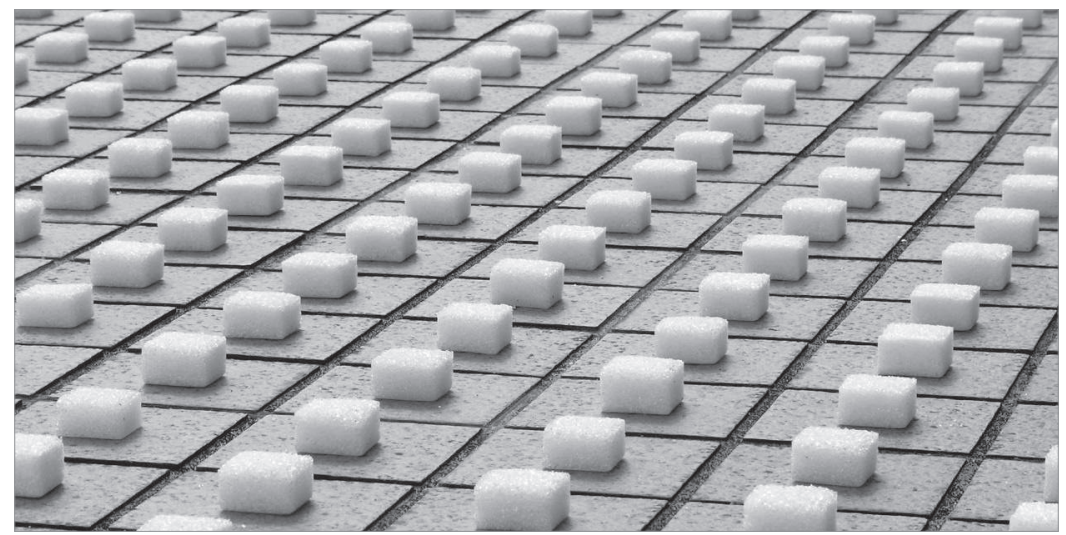

Fig. 8 c Kondō Takahiro, installation Hotaru (uranium et verre concassé), exposition «Architecture et impermanence », Institut franco-japonais du Kansai, avril 2012 ○ Tanabe Mari 田邊真理

morceau d'étoffe, un bol et une cuillère. Le projet s'intitule « Réceptacle de la vie » (Inochi no utsuwa 命のウツワ). Le don dépasse le simple « objet » ${ }^{12}$.

Une autre initiative, lancée par le commissaire Kitagawa Fram 北川フラム avec le soutien de toute l'équipe de la triennale d'Echigo (dans la toute proche région de Niigata), vise à fédérer toutes ces actions de concertation avec la population locale et à apporter par des installations artistiques un soutien moral et un engagement auprès des populations en difficulté. Une multitude de NPO se sont mobilisées pour venir en aide à cette région sinistrée et contribuent à une échelle locale à redonner espoir. L'association Japan Platform fédère à l'échelle nationale l'ensemble de ces initiatives.

12. Nous élaborons, en collaboration avec l'association Patrimoine sans frontières, un projet franco-japonais. L'idée serait de créer des restaurants de la "mémoire " provisoires et mobiles (yatai 屋台) qui circuleraient dans les différents villages de manière à recréer le « lien social » : des lieux de convivialité et des petits événements à une échelle locale. Ce projet est en cours, en collaboration également avec l'association franco-japonaise Caravane qui a déjà réalisé un formidable travail d'aide et de soutien aux populations locales. 


\section{Patrimoine et « lieux de mémoire »}

Les historiens de l'architecture et les spécialistes du patrimoine (IcomosJapan, AIJ, université de Kyoto, etc.) ont lancé de vastes opérations de relevés. En effet, l'idée d'une région et d'une nation se retrouve dans ses monuments, ses paysages célèbres ou ses "lieux de mémoire " (meisho 名 所), signes qui représentent et commémorent une identité collective. Le site de Matsushima 松島, l'un des trois paysages les plus célèbres du Japon, a été relativement épargné par le tsunami car il est en partie protégé par une myriade d'îles. D'autres sites historiques ont été beaucoup plus touchés. Si l'expertise japonaise en matière de reconstruction du patrimoine historique est de très haut niveau, en revanche, force est de constater que la tâche est immense. Se pose également la délicate question de la reconstruction des édifices mineurs qui ne sont pas directement sous la protection du gouvernement. Le témoignage, nous invitant à une réflexion philosophique, de l'architecte-paysagiste Nakamura Yoshio 中村良夫, au lendemain du tsunami, pointait les incohérences du système et le fait que cette catastrophe n'est plus seulement liée à la force de la nature et à son caractère éphémère relevant de l'impermanence (mujo 無常), mais est devenue artificielle, liée à la part de technique et à la part de folie humaine ${ }^{13}$.

Enfin, des philosophes ont organisé une série de conférences sur la "philosophie de la catastrophe " (University of Tokyo, Center for Philosophy, $\mathrm{UTCP})^{14}$. Que restera-t-il de la mémoire de la catastrophe pour les générations futures? Peut-on parler d'une part inconsciente des mémoires collectives: comme si l'homme oubliait le danger, ce danger permanent de la terre prête à trembler. Conserver et archiver la mémoire de la catastrophe pouvant peut-être servir à protéger les générations futures du danger, à protéger des vies... L'enjeu majeur de toutes les actions menées collectivement par les architectes, les urbanistes, les philosophes ou les artistes, sur le patrimoine matériel ou immatériel, étant avant tout celui de la reconstruction du lien social disloqué.

13. Voir : <http://www.mesologiques.com/2011/03/un-petit-rapport-sur-le-seisme-aujapon.html> (26 mars 2011). Je remercie Augustin Berque, qui avait diffusé ce texte au lendemain de la catastrophe.

14. Voir : <http://utcp.c.u-tokyo.ac.jp/events/index.php>. 
Comme le faisait remarquer Michaël Ferrier, lors de la table ronde sur l'après-catastrophe, tenue un an après le 11 mars $^{15}$, cette triple catastrophe fait apparaître des fractures sociales jusqu'alors masquées : fractures au sein de la société japonaise, notamment avec les travailleurs précarisés des centrales nucléaires ; fractures géographiques de la société, entre l'agglomération super-active de Tokyo et les zones rurales qui connaissaient, déjà avant le tsunami, un fort déclin démographique et un vieillissement de la population. En même temps, la catastrophe aura permis de faire émerger des forces de contestation et aussi des forces de solidarité : avec la création d'une multitude de NPO locales, un engagement sur le terrain des populations locales, et aussi chez les jeunes architectes japonais, un formidable élan d'entraide.

Mars 2012

\section{Bibliographie}

BASHŌ (MATSUO Munefusa), 1971

La Sente étroite du Bout-du-monde (Oku no hosomichi 奥の細道), in Journaux de voyage, trad. René Sieffert, Paris, Publications orientalistes de France, coll. Les trois grands du xvile siècle.

\section{FERRIER Michaël, 2012}

Fukushima : récit d'un désastre, Paris, Gallimard.

IGARASHI Tarō (ed.)

五十嵐太郎(著、編集), 2008

Kenchiku to shokubutsu 建築と植物

(Architecture et plantes), Tokyo, Inax.

\section{JEUDY Henri-Pierre 1990}

Le Désir de Catastrophe, Paris, Aubier.

HLADIK Murielle, 2008

Traces et fragments dans l'esthétique japonaise, Wavres, Mardaga.

OGINO Masahiro (dir.) 荻野昌弘 (編)， 2002

Bunka isan no shakaigaku. Rūburu bijutsukan kara genbaku dōmu made 文化遺産の社会学—ルーヴル美術館 から原爆ドームまで (La sociologie des patrimoines culturels. Du Louvre au dôme de Hiroshima), Tokyo, Shin.yōsha 新曜社.

15. Table-ronde "Japon : L'après-désastre ", le 20 mars 2012, organisée par le programme Japon de la FMSH : <http://www.msh-paris.fr/actualites/actualite/article/ japon-lapres-desastre/?cHash $=\mathrm{a} 479 \mathrm{fa} 2114$ \&taillepolice $=0>$. 